Management international

International Management

Gestiòn Internacional

\title{
La localisation des postes d'encadrement dans les entreprises françaises en Chine
}

Localization of Managerial Positions in China-based French

Firms

\section{La localización de puestos directivos en las empresas francesas implantadas en China}

Lingfang Song

Volume 19, numéro 4, été 2015

URI : https://id.erudit.org/iderudit/1043085ar

DOI : https://doi.org/10.7202/1043085ar

Aller au sommaire du numéro

\section{Éditeur(s)}

HEC Montréal

Université Paris Dauphine

\section{ISSN}

1206-1697 (imprimé)

1918-9222 (numérique)

Découvrir la revue

Citer cet article

Song, L. (2015). La localisation des postes d'encadrement dans les entreprises françaises en Chine. Management international / International Management / Gestiòn Internacional, 19(4), 184-198. https://doi.org/10.7202/1043085ar

\section{Résumé de l'article}

La localisation des postes d'encadrement en Chine, que l'on confie désormais à des cadres chinois et non plus à des expatriés, est une tendance de plus en plus prononcée dans les entreprises multinationales. La présente recherche suggère qu'une localisation complète y est peu probable. Deux approches de la localisation sont pratiquées : une approche locale et une approche semi-locale selon le choix prioritaire de trois catégories de cadres chinois: des chinois locaux, des chinois d'outre-mer et des chinois retournés des pays étrangers. Sur la base de l'analyse des pratiques actuelles et des défis rencontrés, des perspectives d'évolution sont proposées.
Tous droits réservés (c) Management international / International Management / Gestión Internacional, 2015
Ce document est protégé par la loi sur le droit d'auteur. L'utilisation des services d'Érudit (y compris la reproduction) est assujettie à sa politique d'utilisation que vous pouvez consulter en ligne.

https://apropos.erudit.org/fr/usagers/politique-dutilisation/ 


\title{
La localisation des postes d'encadrement dans les entreprises françaises en Chine
}

\author{
Localization of Managerial Positions \\ in China-based French Firms
}

\section{La localización de puestos directivos en las empresas francesas implantadas en China}

\author{
LINGFANG SONG \\ ESCEM School of Management, France
}

\begin{abstract}
RÉSUMÉ
La localisation des postes d'encadrement en Chine, que l'on confie désormais à des cadres chinois et non plus à des expatriés, est une tendance de plus en plus prononcée dans les entreprises multinationales. La présente recherche suggère qu'une localisation complète y est peu probable. Deux approches de la localisation sont pratiquées : une approche locale et une approche semi-locale selon le choix prioritaire de trois catégories de cadres chinois: des chinois locaux, des chinois d'outremer et des chinois retournés des pays étrangers. Sur la base de l'analyse des pratiques actuelles et des défis rencontrés, des perspectives d'évolution sont proposées.
\end{abstract}

Mots clés : localisation de l'encadrement, expatrié, Chine, cadres chinois, entreprises multinationales

\begin{abstract}
Management localization (entrusting management positions to Chinese executives, not to foreign expatriates) is an increasing trend in China-based multinationals. This research suggests that a complete localization is unlikely. Two approaches to localization are practiced: a local approach and a semi-local approach, depending on the priority given to the three categories of Chinese executives: local Chinese, overseas Chinese and Chinese returnees when there is a post vacancy. Based on the analysis of current practices and challenges, measures of improvement are put forward. Keywords: localization of managerial positions, expats, China, Chinese managers, multinational firms
\end{abstract}

\begin{abstract}
ReSUMeN
La localización de puestos directivos en China, que se asignan ahora a directivos chinos, y ya no a los expatriados, es una tendencia cada vez más pronunciada en las empresas multinacionales. La presente investigación sugiere que sea poco probable una localización completa. Dos enfoques sobre localización son practicados: un enfoque local y un enfoque semi-local, según la opción prioritaria de tres categorías de directivos chinos: chinos locales, chinos de ultramar, y chinos regresados de países extranjeros. Tomando como base el análisis de las prácticas actuales y los desafíos encontrados, se proponen perspectivas de evolución.

Palabras Claves: localización de puestos directivos, expatriado, China, directivos chinos, empresas multinacionales
\end{abstract}

\begin{abstract}
A l'ère de la mondialisation, les entreprises ne sont plus limitées par les frontières nationales. Les opérations internationales se multiplient. Dans la gestion des opérations d'outre-mer, les équipes managériales des filiales à l'étranger se composent de dirigeants divers de par leur culture et leur expérience internationale. Dans la majorité des cas, deux types de cadres collaborent : des expatriés envoyés du pays investisseur et des cadres locaux du pays d'accueil (Harzing, 2001a). Les premiers comme les seconds constituent de précieuses ressources pour l'entreprise, mais contribuent de manières différentes à la performance. Le recours aux expatriés facilite les transferts de savoir-faire, le contrôle de la filiale, la communication avec le siège et contribue au développement de la carrière des cadres ainsi envoyés (Edström et Galbraith, 1977; Harzing, 2001b; Boyacigiller, 1990; Schaaper et al, 2013). Les cadres locaux, pour leur part, bénéficient d'une connaissance plus intime du marché, ainsi que d'une plus
\end{abstract}

grande facilité de dialogue avec les employés et les clients locaux (Hailey, 1996; Hutchings, 2005; Fayol-Song, 2012). En revanche, par souci de maîtrise des coûts d'encadrement et de rétention des cadres locaux, les entreprises s'efforcent de transférer davantage de postes d'expatrié à ces derniers (Grainger et Nankervis, 2001; Lam \& Yeung, 2010). Décider à quel moment tel ou tel poste d'expatrié doit être transféré à des cadres locaux, dans quelles conditions et à quel rythme, constitue une décision stratégique fondamentale pour les multinationales (Lam \& Yeung, 2010).

Dans le contexte chinois, la localisation des postes d'encadrement est une tendance de plus en plus prononcée chez les entreprises multinationales implantées dans le pays. Une étude effectuée par Hewitt Associates réalisée en 2008 montre que dans les multinationales étudiées en Chine, $21,4 \%$ des postes managériaux de haut 
niveau étaient occupés par des expatriés occidentaux en 2007 contre $24,8 \%$ en 2006 et $30,7 \%$ en 2005 (Hewitt Associates, 2010). Le nombre de chinois retournés des pays étrangers s'est également accru dans les années 2000 (Hober \& Schüssler-Hauri, 2008). Selon PWC Report (2009), la Chine restera un lieu majeur de localisation des postes d'encadrement.

Malgré l'enjeu que représente la localisation de l'encadrement dans la gestion des filiales à l'international, les recherches sur le sujet sont peu nombreuses (Kühlmann et Hutchings, 2010; Lam et Yeung, 2012). Il manque de cadres et de modèles théoriques rigoureusement établis. De plus, les études les plus récentes sont essentiellement réalisées par des groupes de consultants, qui ne présentent qu'un état des lieux sur les postes localisés ou réservés aux cadres expatriés. Elles révèlent rarement les logiques qui sous-tendent les pratiques. De ce fait, la présente contribution se fonde sur une recherche exploratoire qualitative. Elle a pour objectif principal d'identifier et de décrire les différentes pratiques mises en place. Notre intention est de dépasser la simple présentation des chiffres et des pourcentages par une compréhension plus profonde des objectifs et des pratiques développées par les entreprises et leurs conséquences.

En tant que la première étape d'une série des recherches sur le sujet, la présente contribution se focalise sur la question suivante : quelles démarches ces entreprises entreprennentelles dans la localisation des postes managériaux, et quels sont les enjeux? En identifiant les postes localisés et localisables, ainsi que ceux qui pour elles ne le sont pas, nous cherchons à comprendre sur quelle logique les entreprises inscrivent leurs stratégies et pratiques en la matière. Nous souhaitons aussi découvrir quels sont les enjeux et les défis du transfert des postes. L'objectif est de combler une lacune entre les pratiques managériales relatives à la localisation des postes d'encadrement et la compréhension qu'en a la recherche académique aujourd'hui. De plus, les résultats de notre recherche enrichissent notre compréhension sur laquelle se fondent les prochaines étapes de nos travaux, annoncées en conclusion de cet article.

\section{Revue de la littérature}

Le monde des affaires d'aujourd'hui est caractérisé par la diversité, l'incertitude et la complexité. Les entreprises qui s'internationalisent en implantant des filiales à l'étranger sont perpétuellement confrontées, dans la gestion de leurs opérations, l'alternative stratégique fondamentale global vs local, centralisation vs décentralisation, standardisation vs localisation, efficience vs réactivité (Buckley et Ghauri, 2004). Pour concilier de telles tensions, différents remèdes ont été proposés, comme la solution transnationale (Bartlett et Ghoshal, 1998), la solution multifocale (Prahalad et Doz, 1987) et les solutions régio ou géocentrique (Heenan et Permutter, 1979). Tous les modèles proposés ont pour objectif d'établir un équilibre entre les attentes multiples que l'entreprise multinationale perçoit de son environnement.

Dans le domaine de la GRH internationale, la problématique global versus local se traduit concrètement dans le choix stratégique entre l'envoi de cadres expatriés dans les filiales et l'emploi de cadres locaux sur place. Le recours aux expatriés facilite l'intégration globale des filiales alors que l'utilisation des cadres locaux est favorable à la réactivité locale (Schaaper et al, 2012). A chaque entreprise d'établir l'équilibre entre ces deux types de ressources managériales en fonction du contexte où elle se situe. Dans le contexte chinois, caractérisé par une forte distance culturelle et un turnover élevé des cadres locaux, les entreprises multinationales optent de plus en plus pour le transfert des postes d'expatriés au profit des cadres chinois afin de mieux accéder au marché local et en même temps de donner une meilleure perspective d'évolution de carrière aux talents locaux (PWC Report, 2009; Chambre américaine, 15/12/ 2008; Lam et Yeung, 2010). En tant qu'objet de la présente recherche, la localisation des postes d'encadrement est définie comme le remplacement des expatriés par des employés locaux compétents (Huang, 2005; Law et al, 2004; Selmer, 2003). Elle se distingue de la localisation des pratiques managériales, qui, elle concerne l'adaptation des pratiques et approches managériales aux conditions locales (Negandhi et Welge, 1984; Huang, 2005).

Malgré l'intérêt que présente la localisation des postes de management, peu d'entreprises ont réellement transféré tous les postes managériaux à des cadres locaux. Selon Rogers (1999), 5 à 10 pour cent des cadres doivent être des expatriés (pour que l'entreprise maintienne sa caractéristique internationale). L'enquête effectuée par Arthur Andersen (2000) sur 22 entreprises françaises montre également que plus de 47,6 pour cent des répondants pensent que leur entreprise ne pourra jamais se passer d'expatriés; 19 pour cent pensent qu'il s'agit d'une possibilité mais pas dans l'immédiat. Depuis ces constats, datant d'une dizaine d'années, la localisation complète n'est toujours pas réalisée aujourd'hui. Les entreprises multinationales envoient toujours des cadres expatriés dans leurs filiales en Chine (Hewitt, 2010; PWC Report, 2013).

Concernant les postes localisés et localisables, différentes études s'accordent sur certains points, tout en divergeant sur d'autres. Selon le rapport de PWC (2004), les postes les plus localisés dans les années passées concernent la comptabilité, la GRH, la vente et la fabrication. L'enquête de Lau (2005) confirme une localisation fréquente des postes d'encadrement de la GRH et de la vente. 63 pour cent d'entreprises ont nommé des cadres locaux chinois comme responsables de la GRH et 54 pour cent ont mis des cadres locaux comme directeurs des ventes. En revanche, dans des domaines fonctionnels comme la fabrication et la finance où une vision globale de l'entreprise prime sur la perspective locale, la localisation des postes d'expatriés est moins 
avancée : 32 pour cent seulement des entreprises ayant répondu à l'étude de Lau (2005) ont pris les locaux comme responsables de la finance et de la fabrication. Jaussaud et Schaaper (2006) confirment une localisation générale pour les postes d'encadrement de la vente et de la GRH. Parmi les 12 entreprises de leur enquête, 11 ont un cadre local comme directeur commercial sous l'autorité directe du directeur général expatrié. C'est également le cas pour la direction des ressources humaines. Tous les responsables sont des chinois locaux sauf une Singapourienne. La direction de la production est partagée entre les locaux et les expatriés : 4 expatriés et 8 locaux. En revanche dix ans plus tôt, selon Goodall et Warner (1998), la responsabilité de la production et du contrôle qualité était plutôt confiée aux expatriés.

Le poste exclusivement réservé aux expatriés s'avère être celui de la direction générale de la filiale (Goodall et Warner, 1998). Jaussaud et Schaaper (2006) n'identifient aucun transfert sur les 12 cas. Lau (2005) confirme également qu'aucun chinois local dans les filiales de son échantillon n'est en poste de directeur général : 54 pour cent sont des occidentaux, 24 pour cent sont des chinois retournés des pays étrangers, et 22 pour cent sont des chinois d'outre-mer. La direction financière reste aussi massivement affectée à des expatriés (Goodall et Warner, 1998), en particulier au niveau des Holding (Jaussaud et Schaaper, 2006).

Pour résumer, les enquêtes précédentes sont unanimes sur la localisation des fonctions de vente et de GRH, mais présentent des résultats mitigés sur les postes de production; aucune étude ne révèle une localisation de la direction générale. La direction financière est aussi rarement localisée.

Lorsque des postes d'encadrement sont localisés, dans le cas de la Chine, du fait de son contexte historique, politique et économique, les successeurs locaux des cadres expatriés ressortent d'une typologie assez spéciale. Trois types de cadres chinois sont souvent mentionnés dans la littérature (Hulme, 2006; Chan, 2007) : cadres chinois locaux (appelés aussi China Hire), cadres chinois d'outremer (appelés aussi cadres d'origine chinoise), et cadres chinois retournés des pays étrangers.

Les cadres chinois locaux sont ceux qui sont nés en Chine et qui, avant d'être recrutés par l'entreprise multinationale sur place, n'ont pas quitté leur pays pour une période significative (Wong et Law, 1999; Huang, 2005). Les cadres chinois d'outre-mer sont les descendants de chinois qui vivent en dehors de la Chine continentale depuis plus d'une génération (Wang, 1993) comme certains singapouriens, malais, taiwanais et hongkongais. Ils sont de culture chinoise et parlent le chinois comme langue maternelle. Les cadres chinois retournés sont nés et ont grandi sur le continent chinois. Ils sont partis vers les pays développés dans les années de 80 et 90 , le plus souvent pour poursuivre des études de haut niveau. Après l'obtention de diplômes, ils ont débuté leur carrière dans le pays d'accueil puis sont envoyés par leur entreprise depuis le siège dans la filiale en Chine. Ils possèdent soit un passeport soit une carte de résidence étrangers (Chan, 2006).

Par rapport aux chinois locaux et aux expatriés occidentaux, les chinois d'outre-mer et les chinois retournés disposent d'avantages distinctifs liés à leur capacité linguistique, leur connaissance multiculturelle et leur expérience internationale. Dans le contexte chinois de pénurie de talents managériaux locaux, ces deux sortes de cadres, en particulier les chinois retournés, deviennent recherchés par les multinationales opérant en Chine (MFE, 2010; Gross et Connor, 2007).

La localisation des postes d'encadrement en Chine a connu trois phases distinctes (Qiu et Chen, 2005; Lau, 2005). La $1^{\text {ère }}$ phase, du début des années 80 au milieu des années 90, est caractérisée par la domination de la présence des expatriés des pays d'origine dans quasi tous les postes managériaux. La $2^{\text {ème }}$ phase à partir du milieu des années 90 a connu un transfert massif de postes d'expatriés aux chinois d'outre-mer, puis aux chinois retournés des pays étrangers. La $3^{\text {ème }}$ phase, qui est la phase actuelle, est marquée par une croissance du nombre de cadres chinois locaux dans les postes d'encadrement intermédiaire, voire même de haut niveau.

Le marché du travail chinois est caractérisé par la pénurie de cadres locaux qualifiés et le turnover élevé de ce type de personnel (Michael Page China, 2008; Dewhurst et al, 2012). Un des défis majeurs pour les entreprises multinationales s'avère être le développement et la rétention des talents managériaux chinois (PWC Report, 2013; Dodyk, et al, 2012). Selon les enquêtes effectuées par MRIC (2013) et Michael Page China (2008), le turnover est alimenté par le manque d'une perspective claire d'évolution de carrière pour les cadres chinois. En effet, la forte présence des cadres expatriés dans les filiales crée une sorte de plafond de verre vis-à-vis des cadres locaux (Cheung, 2008; Krueger et Ding, 2009). Par conséquent, de plus en plus de cadres locaux quittent les multinationales pour les entreprises domestiques où ils ont plus d'opportunités d'évolution (PWC Report, 2013; Micheal Page China, 2008). La localisation des postes d'expatriés que l'on confie à des cadres locaux est considérée comme une mesure efficace pour développer les compétences locales et la fidélité des salariés chinois envers l'entreprise (PWC report, 2013; Selmer, 2003; Wong \& Law, 1999).

\section{Méthodologie de recherche}

La présente recherche privilège la méthode de l'étude de cas multiple (Eisenhardt, 1989). 12 entreprises françaises ayant des structures en Chine ont été sélectionnées pour notre enquête (Tableau 1). Le choix de l'échantillon est lié au fait que la France est le $3^{\text {ème }}$ investisseur européen en Chine. Les investissements français sont le fait de 
850 entreprises qui comptent environ 1800 implantations distinctes. Elles emploient plus de 250000 salariés dans le pays (MFE, 2011). De plus, les rythmes de progression de l'activité sont rapides (20 à $25 \%$ par an en moyenne), les prises de participations stratégiques se développent, de plus en plus d'entreprises, grandes comme petites, nourrissent des perspectives très ambitieuses dans ce pays (CCIFC, Livre Blanc, 2011). En revanche, comme mentionné précédemment, peu des recherches académiques sur le sujet existent dans la littérature. On dispose encore moins de recherches sur le cas des entreprises françaises. Par ailleurs, le choix des entreprises de la même nationalité nous permet d'écarter les influences culturelles et contextuelles et de se concentrer davantage sur des aspects stratégiques et managériaux.

Dans la collecte de données, nous nous sommes appuyées essentiellement sur la technique des entretiens approfondis semi-directifs. L'examen des documents papier produits par les entreprises dans leur GRH quotidienne, fournis par nos répondants, et la consultation des sites internet des multinationales et leurs filiales chinoises nous ont permis d'avoir une compréhension plus complète et plus précise au travers de la triangulation des données. 31 managers ont été interviewés individuellement ou collectivement. Afin d'avoir une perspective croisée, nous avons intégré dans notre échantillon à la fois des expatriés français (17) et des cadres chinois (14). Au niveau des fonctions, 13 répondants sont dans la direction de la GRH, 8 dans la direction des filiales en Chine, et 10 sont des responsables de service.
La plupart des entretiens ont eu lieu entre 2009 et 2012, essentiellement en Chine et certains en France. Certains répondants ont été interviewés à deux reprises. Les entretiens ont duré une heure trente en moyenne.

Les constats tirés de la littérature existante ainsi que la problématique de la présente recherche nous ont permis d'établir un guide d'entretien en trois sujets thématiques : les types de cadres dans les postes actuels, les démarches de la localisation, et les enjeux (Annexe 1). Afin de faciliter la communication, deux langues d'entretien, en fonction de l'origine des interviewés, le français ou le chinois, ont été employées. Le bilinguisme et la double identité (française et chinoise) que nous possédons facilitent non seulement la communication mais permettent aussi de se rapprocher, en tant que chercheur, des répondants. En effet, la langue choisie pour les entretiens n'est ni «neutre» ni «innocente» (Symon \& Cassell, 2012). Le recours à la langue maternelle du répondant lui permet de s'exprimer plus ouvertement. Les interviewés sont plus disposés à parler librement avec leurs compatriotes.

Le contenu des entretiens a été enregistré par prise de notes écrites et non par magnétophone ou caméra. Le but est d'encourager les interviewés à s'exprimer sans contrainte car le succès d'un entretien approfondi dépend de la capacité de l'enquêteur à faire parler les répondants librement (Kwortnik, 2003). Les gens ont tendance à devenir prudents et mesurés dans leurs réponses, ou décident simplement de se taire lorsqu'ils ressentent la

\section{TABLEAU 1}

Caractéristiques des entreprises enquêtées

\begin{tabular}{l|c|c|c}
\hline & Secteur & Effectif en Chine (personne) & Ancienneté en Chine \\
\hline Cas A & Automobile & 10,000 & +20 ans \\
\hline Cas B & Automobile & 40 & +20 ans \\
\hline Cas C & Energie & 1,000 & +20 ans \\
\hline Cas D & Energie & 250 & +20 ans \\
\hline Cas E & Matériaux de construction & 12,200 & +20 ans \\
\hline Cas F & Conditionnement liquides alimentaires & 350 & +15 ans \\
\hline Cas G & Pompes et équipements & 200 & +10 ans \\
\hline Cas H & Grande distribution & 39,600 & +10 ans \\
\hline Cas I & Grande distribution & 40,000 & +10 ans \\
\hline Cas J & Distribution de jouets et de produits d'enfant & 5 & +10 ans \\
\hline Cas K & Chaine d'hôtels & 16,000 & +10 ans \\
\hline Cas L & Transport aérien & 300 & +40 ans \\
\hline \hline
\end{tabular}


moindre menace comme la présence d'une caméra ou d'un magnétophone.

Dans la restitution des données collectées, nous avons d'abord traduit en français les notes manuscrites prises en chinois au cours des entretiens avec les cadres chinois. Afin d'éviter l'ambigüité ou l'écart de sens produit par la traduction, la technique de la traduction parallèle a été employée : nous avons demandé à un collègue de faire la même traduction, et une comparaison entre les deux versions traduites a ensuite été effectuée pour identifier des écarts et les résoudre, en l'occurrence mineure.

Afin de garder un regard immédiat sur les données collectées, nous avons privilégié la méthode «papier» au cours du traitement et de leur analyse. Nous avons utilisé les logiciels «Excel» et «Word» sous forme de tableaux et de commentaires. En effet, malgré l'intérêt d'un logiciel comme CAQDAS (Computer Aided Qualitative Data Analysis Software), cet outil est susceptible d'éloigner les chercheurs de leurs données collectées (Richards \& Richards, 1994).

Pour traiter les données, nous avons eu recours à la technique du coding. Des mots clés du guide d'entretien ou évoqués par les interviewés de façon directe ou indirecte ont été choisis comme codes ou étiquettes de chaque thème, comme par exemple, «postes localisés», «postes conservés», «types de cadres chinois». Ce dernier code a été ensuite divisé en trois sous-catégories : «cadres locaux», «cadres retournés» et «cadres d'outre-mer».

Dans l'analyse des données, nous avons commencé par l'étude des cas individuels. Cette étape cruciale nous a permis de nous approprier chaque cas à travers une description détaillée des thèmes pour chacun d'eux, notamment les caractéristiques de l'entreprise, les types de cadres chinois recherchés, etc. Cette analyse a pour objectif de produire des résultats qui sont particuliers à chaque cas avant de formuler des constats plus généraux à travers les comparaisons entre les cas (Eisenhardt, 1989). Elle permet de comprendre pourquoi on obtient tel résultat et non d'autres dans un contexte précis. Prenons le cas D comme exemple. Il prévoit une localisation complète sans expatriés d'ici quelques années. L'analyse contextuelle révèle que cette intention est liée à la nature du contrat BOT (Build, Operate, Transfer) signé entre l'entreprise française et le gouvernement local.

Sur la base des résultats révélés par l'analyse des cas individuels, nous avons réalisé ensuite une analyse croisée des douze cas. L'objectif est d'identifier les similitudes et les différences entre les cas (Eisenhardt, 1989). Des comparaisons thématiques constantes (Glaser and Strauss, 1967) nous ont permis de révéler les facteurs présents dans tous les cas, et ceux qui ne sont présents que dans certains d'entre eux. Par une telle analyse, les catégories et les relations découvertes dans chaque cas individuel peuvent évoluer dans un modèle de compréhension plus général et plus cohérent. Par exemple, après une étude transversale de tous les cas concernant le choix prioritaire des successeurs chinois aux expatriés, nous avons pu conceptualiser les pratiques en deux approches de la localisation : une approche locale et une approche semi-locale.

\section{Résultats}

Toutes les entreprises de notre échantillon, excepté le cas $\mathrm{J}$, disposent de cadres français et occidentaux dans leurs structures. Lorsque la perspective d'une localisation complète des postes d'encadrement est évoquée, 8 cas sur 12 revendiquent qu'une localisation complète, que ce soit à court, à moyen ou à long terme, n'est pas envisageable. Les raisons principales sont les exigences du contrôle, les difficultés du transfert des technologies et des savoir-faire, ainsi que le développement des compétences des cadres français dans un contexte international. Les répondants de 2 cas (B et K) sont moins certains sur l'impossibilité d'une localisation complète. Le cas D prévoit une localisation complète à la fin du contrat BOT (Build, Operate, Transfer) en cours. Le cas $\mathbf{J}$ considère que la localisation complète a déjà été réalisée. En effet, dans cette petite structure de 5 personnes sous forme du bureau d'achat, il n'y a pas d'expatriés occidentaux. La directrice, une chinoise retournée de France, a pris la direction du bureau qui était auparavant assurée par un expatrié français.

Pour le cas B, les deux responsables français interviewés ne rejettent pas la possibilité d'une localisation complète. Néanmoins ils ne pensent pas qu'elle se fera à court ou moyen terme. Au moment de l'entretien, notre interlocuteur français était dans le processus du transfert de son poste à un cadre chinois retourné de France. Il considérait ce transfert comme la preuve de la recherche d'une localisation complète car ce poste était celui du plus haut niveau dans la structure. Cela dit, le projet de B en Chine était en sommeil au moment de l'entretien. Il en résulte que beaucoup de postes d'expatriés ont été supprimés. Or, «quand des projets se concrétiseront, il y aura beaucoup plus d'expatriés», a souligné notre interlocuteur. Partant de ce constat, on se demande si le transfert de la direction du bureau est définitif ou plutôt provisoire.

S'agissant du cas D, le cas Build-Operate-Transfer, le transfert de l'opération au partenaire chinois est prévu à la fin du contrat. Après le transfert, il est logique que les responsabilités managériales soient assurées par des cadres chinois. En revanche, cette localisation complète ne sera pas effectuée pendant l'exploitation française. En effet, un quota de quatre expatriés est imputé au budget de l'opération.

Mon adjoint chinois, est tout à fait capable de gérer l'opération mais il ne sera pas le prochain $D G$, nous a informé le DG français. Mon contrat se termine l'an prochain. J'ai déjà proposé ce poste à un collègue français que je connais bien. Mon adjoint chinois n'aura le poste qu'à échéance du contrat BOT, quand l'usine deviendra chinoise à $100 \%$. 


\section{TABLEAU 2}

\section{La présence des expatriés occidentaux et la perspective d'une localisation complète}

\begin{tabular}{|c|c|c|c|c|}
\hline Cas & Secteur & \begin{tabular}{|c|}
$\begin{array}{c}\text { Présence d'expatriés } \\
\text { occidentaux }\end{array}$ \\
\end{tabular} & $\begin{array}{c}\text { Possibilité d'une } \\
\text { localisation complète }\end{array}$ & Remarque \\
\hline $\mathrm{A}$ & Automobile & Oui & Non & \\
\hline $\mathrm{B}$ & Automobile & Oui & Peut-être & Le projet Chine est en sommeil. \\
\hline $\mathrm{C}$ & Energie & Oui & Non & \\
\hline $\mathrm{D}$ & Energie & Oui & Oui & $\begin{array}{l}\text { Le transfert complet n'est prévu } \\
\text { qu'à la fin du contrat BOT. }\end{array}$ \\
\hline $\mathrm{E}$ & $\begin{array}{l}\text { Matériaux de } \\
\text { construction }\end{array}$ & Oui & Non & \\
\hline $\mathrm{F}$ & $\begin{array}{l}\text { Conditionnement } \\
\text { liquides alimentaires }\end{array}$ & Oui & Non & \\
\hline G & Pompes et équipements & Oui & Non & \\
\hline $\mathrm{H}$ & Grande distribution & Oui & Non & \\
\hline $\mathrm{I}$ & Grande distribution & Oui & Non & \\
\hline $\mathrm{J}$ & $\begin{array}{l}\text { Distribution de jouets et } \\
\text { de produits d'enfants }\end{array}$ & Non & Oui & Le transfert complet est considéré achevé. \\
\hline K & Chaine d'hôtels & Oui & Peut-être & L'intention existe mais rien de concret. \\
\hline $\mathrm{L}$ & Transport aérien & Oui & Non & \\
\hline
\end{tabular}

Concernant le cas $\mathrm{K}$, compte tenu de la politique de l'entreprise dans d'autres pays, le directeur délégué de l'opération Chine pense que «il y a une volonté d'avoir une localisation totale dans le futur». L'intention existe mais dans la pratique, rien de concret n'est formalisé. D'après lui, certains postes comme la direction financière et la DRH seront difficiles à localiser. Il n'a pas une idée claire sur comment et quand se fera la localisation en Chine.

Le tableau 2 indique la présence des expatriés ainsi que la probabilité d'une localisation complète dans chaque entreprise.

De la perspective chinoise, notre enquête montre que tous les interviewés pensent qu'une entreprise sans présence française ne serait pas souhaitable. Les cadres chinois mettent l'accent sur l'image française concrétisée par la présence physique d'expatriés. Cette présence non seulement rassure les clients chinois sur la qualité des produits et le prestige de la marque mais aussi crée des opportunités d'apprentissage pour les cadres chinois. Cet avis est partagé par la directrice chinoise du cas $J$ où il n'y avait pas d'expatriés lors de l'entretien. «Le bureau fonctionne bien sans expatriés jusqu'aujourd'hui. Mais je pense que la présence d'un occidental pourrait rendre notre structure française plus tangible. Je suis partante d'intégrer un acheteur français dans mon équipe». Le nouveau directeur chinois du bureau de représentation du cas B a aussi exprimé son souhait de garder un ou deux expatriés français dans les postes commerciaux et techniques. "C'est plus crédible aux yeux de nos clients chinois. Une entreprise française sans français, ce n'est pas très logique» a-t-il dit. Un autre répondant a montré son envie d'apprentissage en travaillant avec des collègues français au quotidien : "J'ai choisi d'être employé par une entreprise occidentale car je peux travailler avec des supérieurs et des collègues français. J'ai envie d'apprendre leur façon de travailler. C'est aussi un plaisir de pouvoir parler le français tous les jours ».

Notre enquête constate que la présence des cadres expatriés dans les filiales est nécessaire non seulement du point de vue français mais aussi de celui des chinois. Il est peu probable qu'une localisation complète soit effectuée. Parmi les douze entreprises, seulement une est gérée sans expatriés actuellement, mais cette situation pourrait changer dans l'avenir. Trois autres cas indiquent l'éventualité d'une localisation complète mais l'analyse de leur contexte respectif ne confirme pas vraiment cette éventualité. De ce fait, nous cherchons à préciser quels sont les postes plutôt réservés aux expatriés français, et quels postes sont localisés ou à localiser en les transférant à des cadres locaux.

Un consensus partagé par tous les interviewés est que les postes les plus sensibles, qualifiés de stratégiques, sont 
des postes clés de haute responsabilité, qui ont un impact décisif sur la performance de l'opération. Il s'agit de la direction générale des opérations en Chine, notamment la direction des filiales, et celle du siège régional Chine ou de la holding le cas échéant. Ces postes sont systématiquement occupés par des cadres expatriés français. Aucun transfert de ces postes n'est envisagé.

A l'égard des postes de supervision concernant essentiellement la direction d'unités opérationnelles (comme directeur d'usine ou directeur de magasin) et la direction financière dans l'unité opérationnelles, quatre constats sont établis. Premièrement ces postes sont occupés essentiellement par des cadres expatriés français. En effet, à travers ces postes, l'entreprise surveille non seulement le fonctionnement quotidien mais aussi la situation financière de ses unités. Deuxièmement, la localisation de la direction d'unités opérationnelles est plus avancée que la direction financière. Entre les deux postes, la direction financière est considérée comme plus critique. Lorsque poste de direction financière est transféré à un local, la supervision de ce poste est parfois assurée par le directeur général expatrié (cas F, H, I). Troisièmement, la présence forte de chinois d'outre-mer et de chinois retournés des pays étrangers est observée dans les postes de supervision. Quatrièmement, la localisation des postes de supervision se réalise plus facilement lorsque l'entreprise dispose d'un siège régional ou d'une holding dans le pays, qui exerce le contrôle sur les unités. Enfin, la situation dans une coentreprise est un cas à part. L'attribution des postes de supervision dépend du contrat de partenariat (cas A) qui précise les titulaires de tous les postes managériaux.

Concernant les postes fonctionnels, il s'agit de ceux de la direction des départements et des services. Notre enquête indique que la fonction de la GRH est localisée dans quasiment toutes les entreprises sauf pour le cas L qui est encore dans la recherche d'un successeur local qualifié. Dans la plupart des cas, ce sont des chinois locaux qui assurent la fonction. Quelques chinois d'outre-mer ou retournés sont aussi présents (cas E, J, K). L'argument soutenant la localisation du poste est que pour bien gérer le personnel local, il faut quelqu'un qui le comprenne. Il est difficile pour les occidentaux de comprendre de manière précise la mentalité et les comportements des locaux ainsi que la subtilité du droit social et des règlements locaux du travail. A cela s'ajoute tout simplement le problème de la langue chinoise dans la communication et la négociation avec les salariés locaux. "C'est du chinois», plaisantait un expatrié.

Toutes les entreprises enquêtées ne disposent pas de service marketing comme les cas $\mathrm{A}, \mathrm{D}$, et $\mathrm{J}$. La frontière entre la fonction du marketing et celle de la vente reste assez floue. Dans certaines entreprises, le marketing s'oriente vers l'activité de la vente alors que d'autres entreprises prennent le concept marketing dans son sens large. Il incorpore une dimension stratégique qui influence la politique et la stratégie générale des opérations en Chine.

Dans le cas où le marketing dépasse la simple fonction de vente et incorpore une dimension stratégique, il reste dans les mains d'un expatrié français. Concernant le transfert $\mathrm{du}$ poste de directeur marketing, deux avis coexistent. Lopinion favorable à la localisation souligne que les locaux ont l'avantage de leurs compétences linguistiques et culturelles. Leurs réseaux de relations leur permettent de mieux exercer les activités de marketing dans le marché local. En revanche, l'opinion opposée souligne que le marketing reste un concept nouveau en Chine. Les locaux n'ont pas encore acquis suffisamment les compétences en marketing. "Beaucoup de gens pensent que le marketing est égal à la vente», a souligné un directeur expatrié. La dimension stratégique incorporée dans le marketing est considérée comme très importante par plusieurs de nos répondants. Il est alors plus prudent de conserver la direction du marketing entre les mains de cadres expatriés et d'intégrer des locaux dans l'équipe de marketing pour des postes de soutien, comme directeur délégué de marketing par exemple. $\mathrm{Si}$ au contraire la fonction de marketing s'appuie essentiellement sur la vente dans le marché local, le poste est souvent confié à un local (cas E, H, et I). Lorsque la vente ne se limite pas au marché chinois continental mais s'étend à la zone Grande Chine voire même à la zone AsiePacifique, l'entreprise confie parfois la responsabilité à un chinois d'outre-mer (cas E et F).

La fonction d'achat est souvent assurée par un local si l'approvisionnement n'est que pour les opérations locales. $\mathrm{Si}$ l'achat est pour approvisionner toutes les opérations de l'entreprise, y compris hors de Chine, la direction des achats est confiée à un français par souci d'efficacité de la communication avec le siège et les autres unités du groupe et d'une vision globale. La direction des systèmes d'information est occupée soit par des français soit par des chinois d'outre-mer ou retournés. Les locaux ne sont présents que dans les unités opérationnelles. Ce fait est lié avec la confidentialité des informations de l'entreprise.

Dans les entreprises industrielles, la direction de la production est occupée majoritairement par des expatriés. Les postes de direction de la maintenance et du contrôle de la qualité liés à la fabrication sont aussi souvent occupés par les cadres français. Il existe plusieurs explications. D'abord, la fonction production est critique dans le transfert des connaissances techniques et technologiques; deuxièmement, la compétence locale manque encore de maturité pour la fabrication des produits de haute technologie et de haut de gamme; enfin, le maintien du poste dans les mains d'un expatrié est un bon moyen d'éviter la divulgation de secrets technologiques.

Il existe en outre d'autres postes fonctionnels spécifiques à chaque entreprise, comme la direction de la $\mathrm{R} \& \mathrm{D}$, la direction des relations publiques, celle de l'expansion et du développement, le process management, le coaching, 
TABLEAU 3

Bilan des titulaires de la direction des postes fonctionnels

\begin{tabular}{l|l|l|l}
\hline \multicolumn{1}{c|}{ Direction } & \multicolumn{1}{|c|}{ Cadres français } & \multicolumn{1}{c}{$\begin{array}{c}\text { Chinois d'outre-mer } \\
\text { chinois retournés }\end{array}$} & \multicolumn{1}{c}{ Chinois locaux } \\
\hline GRH & Rare & Minoritaire & Majoritaire \\
\hline Marketing & $\begin{array}{l}\text { Dans la fonction du marketing } \\
\text { stratégique }\end{array}$ & $\begin{array}{l}\text { Dans la fonction du marketing } \\
\text { régional }\end{array}$ & $\begin{array}{l}\text { Dans la fonction de la vente } \\
\text { locale }\end{array}$ \\
\hline Achat & $\begin{array}{l}\text { Si l'achat est pour l'ensemble } \\
\text { des opérations de l'entreprise }\end{array}$ & $\begin{array}{l}\text { Si l'achat couvre la zone } \\
\text { asiatique }\end{array}$ & Si l'achat est local \\
\hline $\begin{array}{l}\text { Production/ } \\
\text { Contrôle de qualité / } \\
\text { Maintenance }\end{array}$ & Majoritaire & Minoritaire & Minoritaire \\
\hline $\begin{array}{l}\text { Système } \\
\text { d'information }\end{array}$ & $\begin{array}{l}\text { Majoritaire surtout à la holding } \\
\text { et au siège régional }\end{array}$ & Majoritaire & $\begin{array}{l}\text { Essentiellement dans des unités } \\
\text { opérationnelles }\end{array}$ \\
\hline \hline
\end{tabular}

etc. Les responsables de ces services sont majoritairement français, chinois d'outre-mer ou retournés, sauf ceux des relations publiques et de l'expansion et du développement. Ces derniers sont pris par les chinois locaux à cause de leurs réseaux, indispensables dans ces postes. Le tableau 3 synthétise les types de cadres dans les postes fonctionnels.

A l'égard du management de première ligne soit le niveau juste au-dessus des employés non-cadres comme les chefs de rayon ou les chefs d'équipe, une localisation massive des postes par transfert à des salariés chinois locaux est constatée. C'est ainsi le cas pour des postes opérationnels en comptabilité, dans les centres d'appels, etc.

Nous avons posé la question suivante : parmi les trois types de cadres chinois (chinois locaux, chinois retournés et chinois d'outre-mer), quel(s) types de cadre les entreprises préfèrent comme bénéficiaires de la localisation? A cet égard, les entreprises enquêtées divergent. Cinq cas A, B, $\mathrm{D}, \mathrm{H}$, et I, mettent les chinois locaux en position prioritaire. Les citations suivantes relèvent les raisons de leur choix :

On ne voit pas d'intérêt à employer les chinois d'outremer. Ils parlent peut-être mieux l'anglais mais à part $c ̧ a$, ils n'ont pas d'autres avantages par rapport aux chinois locaux. En plus, ils sont plus chers et ne peuvent pas rester en permanence sur place, nous a affirmé le directeur d'usine du cas A.

J'ai beaucoup entendu parler, parmi mes confrères d'autres entreprises, des conflits entre les retournés et les locaux, a exprimé l'ancien DRG expatrié du cas $\mathrm{H}$. Pour faire rentrer les retournés en Chine, il faut leur donner des conditions de travail attirantes, forcément meilleures que celles du contrat local. Les locaux se sentent mal de voir que leurs compatriotes ont plus d'avantages qu'eux. Le sentiment d'injustice n'est pas bon pour les motiver ni pour les fidéliser. Du coup, chez nous, on ne cherche que des chinois locaux. C'est plus simple.

Le directeur de production expatrié du cas B souligne que, aujourd'hui, il y a beaucoup moins d'intérêt d'avoir des chinois d'outre-mer ou retournés qu'il y a dix ans car les profils des locaux se sont beaucoup améliorés. Ils sont plus professionnels et parlent aussi mieux l'anglais.

Un des intérêts principaux de la localisation est d'avoir des connaissances intimes sur le marché local. Les chinois d'outre-mer sont plutôt étrangers que locaux à part qu'ils parlent le chinois. Ils sont nés et ont grandi en dehors de la Chine continentale. Leur connaissance du marché est assez limitée. De plus, ils n'ont pas de réseaux professionnels comme les locaux, nous a expliqué le DRH local du cas I. Il est vrai que les chinois retournés sont beaucoup plus chinois que les chinois d'outre-mer à cause de leur lien étroit avec le pays et leur vécu sur place. Mais après une absence de longue durée du pays, souvent plus de 10 ans voire même plus de vingt ans, ils sont plus ou moins déconnectés $d u$ marché d'aujourd'hui. Le marché chinois évolue très vite. On doit le suivre de très près constamment.

Pour résumer, il existe cinq raisons principales qui orientent les entreprises vers les chinois locaux :

Le coût économique : les chinois d'outre-mer ou retournés sont plus chers.

- La stabilité : les chinois d'outre-mer ou retournés ne restent en Chine que pour une durée limitée. 
- Les connaissances locales : les chinois d'outre-mer ou retournés sont moins connectés au marché local.

- Le conflit entre les cadres chinois : les chinois locaux n'acceptent pas d'être traités inférieurement aux chinois d'outre-mer ou retournés.

- L'amélioration de compétence locale : l'écart de compétence entre les trois types de cadres se réduit de façon continue.

Cela-dit, toutes les entreprises n'ont pas un avis favorable aux cadres locaux. Sept d'entre elles (cas C, $\mathrm{E}, \mathrm{F}, \mathrm{G}, \mathrm{J}, \mathrm{K}$ et L) montrent une attitude réservée sur la compétence locale. Les opinions citées ci-dessous révèlent que, par rapport aux chinois locaux, les chinois retournés ou d'outre-mer se distinguent dans leur professionnalisme, leur ouverture d'esprit et leur fidélité à l'entreprise.

Ce n'est pas par hasard que nous préférons les chinois non continentaux car ils sont plus performants en termes de professionnalisme et de compétences managériales et linguistiques. Ils sont aussi plus stables que les locaux, sachant que le taux de turnover est très élevé chez les locaux. (Cas L)

Les locaux n'ont pas encore fini leur phase d'apprentissage. Leur vision n'est pas assez large. Leur mentalité est aussi très chinoise. Il est trop tôt pour leurs confier de hautes responsabilités. Les chinois d'outre-mer ou retournés ont un avantage là-dessus à cause de leurs expériences internationales. (Cas G)

La priorité que l'entreprise accorde dans le choix du successeur à un poste occupé jusqu'alors par un expatrié a des impacts sur les ressentiments et la satisfaction des cadres chinois locaux. Si les locaux acceptent plutôt bien le fait que les postes stratégiques et de contrôle soient essentiellement dans les mains des expatriés, ils trouvent injustifié que les chinois d'outre-mer ou retournés soient prioritaires dans la localisation des postes. Plusieurs témoins ont exprimé leur incompréhension à ce sujet.

Nous avons le sentiment d'être des cadres de troisième classe après les français et les chinois non-résidents. Le plafond de verre a une double épaisseur chez nous. Je me demande maintenant s'il n'est pas plus intéressant de travailler dans une entreprise chinoise. Au moins, il n'existe pas ce genre de plafond de verre. (Cas L).

Nous sommes souvent critiqués d'être infidèles envers l'entreprise. Si nous ne voyons pas clairement la perspective d'évolution, pourquoi y rester? (Cas E).

Dans mon entreprise, nous ne connaissons pas vraiment la politique de la localisation. Il n'y a jamais eu de communications officielles à ce propos. Nous essayons de comprendre par observation ce qui se passe réellement. Chez nous, il y a très peu de chinois d'outre-mer ou retournés. C'est plutôt un bon signe pour nous les locaux. Mais on ne sait pas si c'est voulu ou seulement par hasard. (Cas A).

Le dernier propos souligne l'importance d'une communication explicite de la stratégie de l'entreprise en matière de localisation des postes d'encadrement. Si les cadres locaux sont considérés comme prioritaires dans la localisation, ce choix peut être motivant dans la rétention des talents locaux.

\section{Discussion}

Les résultats de notre recherche concordent avec le constat tiré des études existantes : une localisation complète sans expatriés est peu probable. Ce constat a été confirmé et plébiscité à la fois par les répondants français et par les répondants chinois de notre enquête.

Au lieu d'analyser la localisation poste par poste comme dans la plupart des études précédentes, nous avons classifié les postes en quatre catégories : postes stratégiques, postes de supervision, postes fonctionnels et postes de première ligne. Cette classification nous a permis de mieux situer le niveau du management où se passe la localisation. En général, les entreprises réservent les postes stratégiques aux expatriés, et sont aussi moins enclines à transférer les postes de contrôle aux chinois locaux. Différents cas de figures sont présents dans la localisation des postes fonctionnels selon la nature des postes. Une localisation complète, enfin, est réalisée dans les postes de première ligne. En accord avec certaines études précédentes (Jaussaud et Schaaper, 2006; PWC, 2004), notre recherche interroge néanmoins le constat de certains auteurs selon lequel dans la $3^{\text {ème }}$ phase de la localisation, celle d'aujourd'hui, la localisation toucherait les postes des plus hauts niveaux (Qiu et Chen, 2005). En effet, la localisation des postes stratégiques et de contrôle n'est pas selon nous encore d'actualité, au moins dans les entreprises françaises impliquées dans notre recherche.

Les études précédentes se focalisent généralement sur deux types de dirigeants dans la gestion des filiales : expatriés occidentaux et cadres chinois. Tenant compte du contexte particulier du pays, nous avons distingué les cadres chinois en trois catégories dans notre enquête : chinois locaux, chinois retournés et chinois d'outre-mer. Cette catégorisation nous a permis d'établir deux approches de la localisation pratiquées dans les entreprises selon le choix prioritaire accordé à trois types de cadres chinois : une approche locale et une approche semi-locale (Figure 1).

Avec l'approche locale, l'entreprise met les chinois locaux en position prioritaire comme bénéficiaires de la localisation lorsqu'un poste d'expatrié fait l'objet d'un transfert. Si aucune compétence locale n'est identifiée, l'entreprise élargit son champ de recherche aux chinois retournés ou d'outre-mer. Dans ces entreprises, les chinois locaux restent majoritaires par rapport aux cadres chinois retournés ou d'outre-mer. 


\section{FIGURE 1}

\section{Deux approches de localisation}

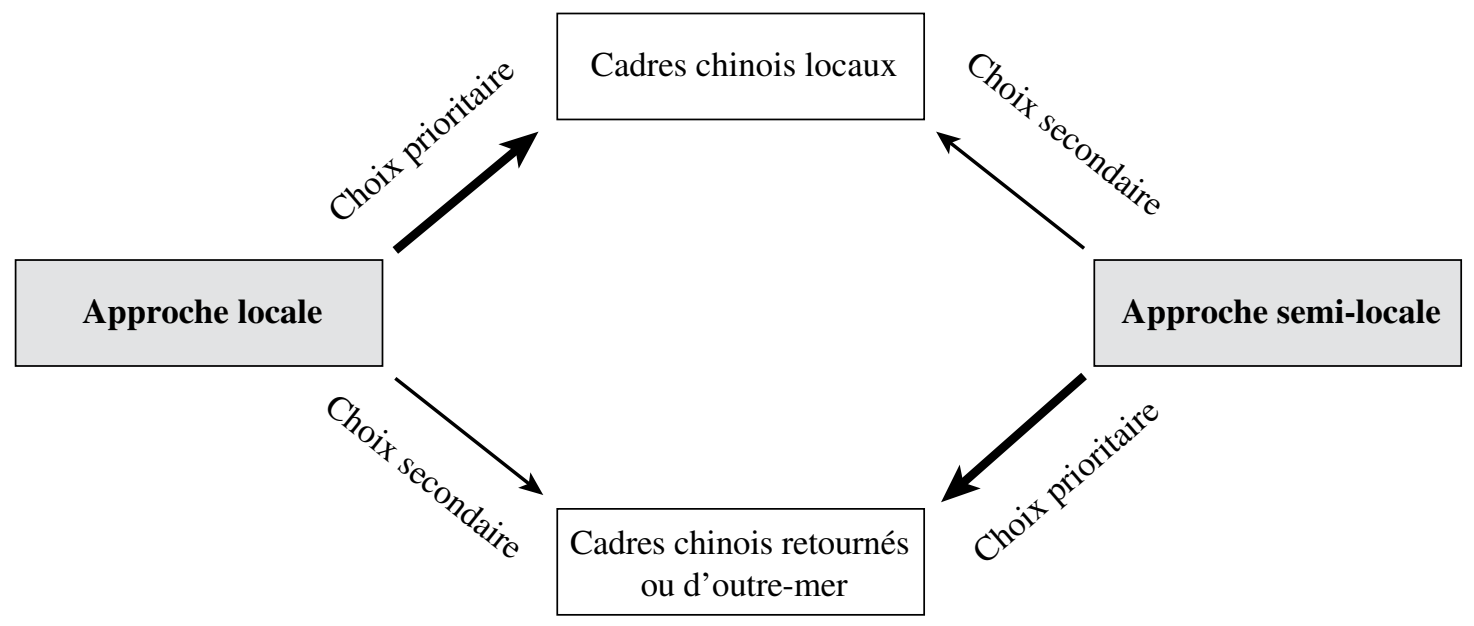

Dans la démarche semi-locale, les entreprises accordent la priorité aux cadres chinois retournés ou d'outre-mer. Elles choisissent de les placer dans la plupart des postes de direction, surtout au niveau de la holding, du siège régional et du bureau de représentation. Les locaux sont placés dans les postes d'importance inférieure.

L'approche de la localisation pratiquée par l'entreprise a un impact non négligeable sur la satisfaction et la rétention des cadres locaux. La forte présence des expatriés occidentaux dans les postes stratégiques et du contrôle crée une sorte de plafond de verre pour les locaux. Dans les entreprises pratiquant l'approche de la localisation semi-locale, le choix prioritaire des chinois retournés ou d'outre-mer crée une deuxième couche du plafond de verre. L'existence de ces plafonds obscurcit la perspective d'évolution de carrière chez les locaux; elle constitue un facteur susceptible de déclencher le départ volontaire des talents locaux vers des entreprises chinoises.

Cette situation crée un dilemme pour les entreprises multinationales dans la gestion de leurs filiales en Chine. D'un côté, la présence des expatriés dans les postes stratégiques et du contrôle est nécessaire, et de l'autre, ce plafond de verre ne facilite pas la rétention des cadres locaux. Ces derniers ont besoin d'espace pour évoluer. L'entreprise est censée trouver des voies de développement pour que les hauts potentiels locaux puissent accéder un jour aux plus hautes responsabilités.

\section{IMPLICATIONS MANAGÉRIALES}

Notre recherche révèle la coexistence de quatre types des cadres dans la gestion des filiales en Chine : des expatriés, des chinois locaux, des chinois retournés et des chinois d'outre-mer. Ils sont complémentaires par leurs profils, leurs expériences et leurs compétences. En raison des nécessités de contrôle, de transfert de savoir-faire et de l'image française, il est peu probable qu'une localisation complète, c'est-à-dire sans expatriés, ait lieu dans le court, le moyen, voire même le long terme. De ce fait, une localisation à 100 pour cent ne doit pas devenir un objectif à poursuivre dans la démarche de la localisation. C'est aussi le cas pour la présence des cadres chinois retournés ou d'outre-mer. Ils sont présents actuellement et continueront à être recherchés tant qu'ils disposeront des compétences encore trop rares chez les cadres locaux.

Afin de pouvoir répondre simultanément aux besoins multiples des filiales en Chine (contrôle, image, apprentissage, communication, développement et rétention des cadres locaux), nous proposons quelques bonnes pratiques susceptibles d'être utiles dans le processus de la localisation des postes d'encadrement. L'objectif n'est pas de confier tous les postes aux chinois locaux en supprimant la présence des autres types de cadre. Ce qui importe est de briser le plafond de verre en éliminant la catégorisation systématique des cadres dans l'attribution des postes et des avantages. Voici les trois mesures concrètes dans la gestion des postes-clés :

- Premièrement, intégration de tous types de cadres dans les différents niveaux hiérarchiques. Concrètement, un chinois retourné ou d'outre-mer pourrait être sous la direction d'un cadre local, ou un français être subordonné à un chinois retourné si les seconds ont un meilleur profil et sont plus performants. 
- Deuxièmement, transparence sur la politique de la localisation. L'importance est de communiquer à tous les cadres de façon officielle régulière sur la politique de localisation des postes d'encadrement et les mesures mises en place en soulignant les compétences recherchées.

- Enfin, troisièmement, développement des compétences des cadres locaux, y compris par un programme d'internationalisation. Le terme «internationalisation» désigne l'implication de certains cadres locaux dans l'ensemble des opérations de l'entreprise. Lorsqu'un cadre à haut potentiel est identifié, l'entreprise devrait lui confier la responsabilité d'un poste au siège, ou dans d'autres unités hors de Chine, pour une durée relativement longue. Cette expérience lui permet de se familiariser de manière plus profonde avec les rouages de l'entreprise, d'être plongé dans la culture organisationnelle qui la caractérise, et d'établir des réseaux professionnels. La mutation est aussi favorable au développement d'une vision plus large sur l'ensemble des activités de l'entreprise. Au cours de ces expériences, un attachement fort à l'entreprise peut être créé chez l'individu. Ce lien est un facteur déterminant dans la rétention des talents managériaux. Dans cette perspective, les cadres chinois internationalisés deviendront une ressource stratégique compétitive, capable d'occuper des postes de haut niveau, stratégiques ou de contrôle.

La Figure 2 résume les résultats de la présente recherche ainsi que les recommandations proposées en mettant en liaison les pratiques actuelles, les pistes d'évolution et l'objectif à atteindre. Les pratiques existantes ont tendance à créer des difficultés dans le développement et la rétention des talents locaux. Les pistes d'amélioration identifiées par la présente recherche ont pour objectif de faire évoluer les pratiques actuelles et de mieux répondre simultanément aux besoins multiples.

\section{Conclusion}

Quelles démarches les multinationales implantées en Chine entreprennent-elles dans la localisation des postes managériaux, et quels sont les enjeux ? Pour répondre à cette question de recherche, nous avons étudié les politiques et les pratiques mises en place par douze entreprises françaises en Chine. Les apports de notre recherche sont principalement de quatre ordres. D'abord, en employant une démarche académique, elle a en partie comblé le fossé existant entre les pratiques managériales en la matière et la compréhension qu'en ont aujourd'hui les chercheurs. Deuxièmement, une perspective locale a été intégrée à notre enquête, par l'interrogation de cadres chinois et non seulement de cadres expatriés, pour que notre analyse soit plus complète. Troisièmement, la classification des cadres chinois en trois catégories (chinois locaux, chinois d'outre-mer, chinois retournés) nous permet d'avoir une compréhension plus détaillée et plus subtile sur la démarche de localisation des postes d'encadrement. Quatrièmement, sur la base de nos résultats, des recommandations managériales ont été formulées à l'intention des dirigeants des entreprises multinationales dans leur démarche de localisation.

FIGURE 2

\section{Pratiques, évolution, objectif}

\begin{tabular}{|c|c|c|}
\hline Pratiques actuelles & Pistes d'amélioration & $\begin{array}{l}\text { Objectif : Satisfaction } \\
\text { de besoins multiples }\end{array}$ \\
\hline $\begin{array}{l}\text { Coexistence de } 4 \text { types } \\
\text { de cadres : } \\
\text { - expatriés : } \\
\text { postes stratégiques } \\
\text { et de contrôle } \\
\text { - } 3 \text { types de chinois : } \\
\text { partages des postes } \\
\text { fonctionnels } \\
\text { Deux approches : } \\
\text { - approche semi-locale } \\
\text { - approche locale }\end{array}$ & $\begin{array}{l}\text { - Intégration de } 4 \text { types } \\
\text { de cadres dans tous } \\
\text { niveaux managériaux } \\
\text { - Transparence sur la } \\
\text { politique de la localisation } \\
\text { - Internationalisation } \\
\text { des cadres locaux }\end{array}$ & $\begin{array}{l}\text { - Contrôle } \\
\text { - Apprentissage } \\
\text { - Image } \\
\text { - Développement } \\
\text { - Rétention }\end{array}$ \\
\hline
\end{tabular}


Le transfert des postes managériaux aux cadres locaux est un processus multidimensionnel qui exige une coordination entre trois acteurs principaux engagés dans les différentes étapes du processus : la direction de la multinationale, les cadres expatriés et les cadres locaux. En tant que première étape de notre recherche sur le processus de localisation des postes d'encadrement, cette contribution se focalise sur la nature des postes localisés et localisables, et sur les bénéficiaires de la localisation. Nos recherches futures ont pour objectif de découvrir les facteurs influençant les démarches adoptées ainsi que d'identifier les aspects critiques et les rôles interdépendants de trois acteurs principaux dans le processus de la localisation des postes d'encadrement.

Notre recherche s'inscrit dans une démarche managériale plutôt que culturaliste. Elle a choisi d'impliquer uniquement des entreprises françaises. Néanmoins, conscients des influences significatives de l'origine de l'entreprise sur le développement des stratégies organisationnelles et la mise en œuvre des pratiques managériales, nous envisageons, dans une nouvelle recherche, d'intégrer des entreprises d'autres nationalités afin d'obtenir une vision plus large et complète sur la localisation du management.

\section{Bibliographie}

Andersen, Arthur (2000). «Les Ressources Humaines dans les entreprises franco-chinoises », Internal report communicated by Arthur Andersen Company.

Bartlett, Christopher A.; Ghoshal, S. (1998). Managing Across Borders. The Transnational Solution (2 ${ }^{\text {ème }}$ Ed.), Harvard Business School Press.

BoyacigILLER, N. (1990). «The role of expatriates in the management of interdependence, complexity and risk in multinational corporations», Journal of International Business Studies, Vol. 21, №3, p. 357-81.

Buckley, P. J.; GHAuri, P. N. (2004). «Globalisation, economic geography and the strategy of multiational enterprises», Journal of International Business Studies, Vol. 3, $\mathrm{N}^{\circ} 2$, p. 81-98.

CCIFC, Livre Blanc. (2011). «Le défi des investissements français en Chine», Disponible sur http : //www.ambafrance-cn.org/ IMG/pdf/livre-blanc.pdf, [Consulté le 27/06/2014]

Chambre américaine (15/12/2008). «2008 Hewitt Expatriate, China Hire \& Returnee Compensation and Benefits Study», Disponible sur http : //www.amcham-shanghai.org/ AmChamPortal/MCMS/Presentation/Template/Content. aspx ? Type $=32 \&$ Guid $=\% 7$ BC443FD99-10C8-4ACF-813DE51D07D70BB3\%7D, [Consulté le 28/10/2013]

CHAN, Y. (2006). «THE LIMITS OF BRAIN-CIRCULATION : Chinese returnees and technological development in Beijing», Disponible sur http : //www.cctr.ust.hk/materials/working papers/WorkingPaper15.pdf, [Consulté le 12/02/2009]

Chan, J. (2007). «Local Hire», Amcham China Brief, February 2007.
Cheung, L.L.W. (2008). «Let the "other" speak for itself : Understanding Chinese employees from their own perspectives», Critical perspectives on international business, Vol. 4, $\mathrm{N}^{\circ}$ 2/3, p. 277-306.

Dewhurst, M.; Pettigrew, M.; Srinivasan, R. (2012). «How multinationals can attract the talent they need», June 2012, Disponible sur http : //www.mckinsey.com/insights/ organization/how_multinationals_can_attract_the_talent_ they_need, [Consulté le 28/11/2013]

Dodyk, P; Richardson, A.; Wu, M. (2012). «Talent Management at Multinational Firms in China»,Knowledge@Wharton, January 03, 2012, Disponible sur http : //knowledge.wharton. upenn.edu/printer_friendly.cfm ?articleid=2903, [Consulté le 28/10/2013]

Edström, A.; Galbraith, J.R. (1977). «Transfer of Managers as a Coordination and Control Strategy in Multinational Organizations», Admistrative Science Quarterly, Vol.22, p. 248-263.

EIsEnhardt, K. M. (1989). «Building Theories from Case Study Research, Academy of Management», The Academy of Management Review, Vol. 14, $\mathrm{N}^{\circ}$ 4, p. 532-550.

FAYOL-SONG, L. (2012). «Internationalization of Chinese Executives», Global Journal of Business Research, Vol. 6, $\mathrm{N}^{\circ} 1$, p. 47-54.

Glaser, B.G.; Strauss, A. (1967). Discovery of Grounded Theory: Strategies for Qualitative Research, Sociology Press.

Goodall, K.; Warner, M. (1998). «HRM Dilemmas in China : The Case of Foreign-Invested Enterprises in Shanghai», Asia Pacific Business Review, Vol. 4, N 4, p.1-21.

Grainger, R.J.; NANKervis, A.R. (2001), «Expatriation practices in the global business environment», Research and Practice in Human Resource Management, Vol. 9, $\mathrm{N}^{\circ}$ 2, p. 77-92.

Gross, A.; Connor, A. (2007). «Managing Chinese Returnees», Mobility Magazine, Disponible sur http : //www.pacificbridge. com/publications/managing-chinese-returnees/, [Consulté le 08/03/2009]

Hailey, J. (1996). «Breaking through the glass ceiling», People Management, Vol. 2, №14, p. 32-46.

Harzing, A.W. (2001a). «Who's in charge? An empirical study of executive staffing practices in foreign subsidiaries », Human Resource Management, Vol. 40, N² , p.139-158.

Harzing, A.W. (2001b). "An analysis of the functions of international transfers of managers in MNCs», Employee Relations, Vol. 23, № 6 , p. 581-98.

Heenan, D. A.; Perlmutter, H. V. (1979). «Multinational Organizational Development : A Social Architectural Approach», Reading, MA : Addison-Wesley.

HewitT. (2010). «Hewitt 2010 Expatriate C\&B Study », Disponible sur http : //www.amcham-shanghai.org/amchamportal/ infovault_library/2010/2010_Expatriate_Returnee_and_ China_Hired_Foreigner_Compensation_and_Benefits_ Survey.pdf. [Consulté le 27/06/2014].

Horber, B.; Schüssler-Hauri, B. (2008). «La Chine attire de la main d'œuvre de l'étranger », l'enquête de Hewitt Associates, 2008-02-21, Disponible sur http : //www.hewittt.ch, [Consulté le $12 / 03 / 2009$ ] 
Huang, S. (2005). «Management Localization and Performance of MNCs in China : A Contingent Resource-based view». Disponible sur http : //www.library.ln.edu.hk/ethesis/ huang_051114.pdf. [Consulté le 20/01/2009]

Hulme, V. A. (2006). «Short Staffed», The China Business Review, Vol.33, $\mathrm{N}^{\circ} 2$, p.18-23.

Hutchings, K. (2005). «Koalas in the land of the pandas : reviewing Australian expatriates' China preparation», International Journal of Human Resource Management, Vol. 16, $\mathrm{N}^{\circ}$ 4, p. 553-66.

JAUSSAUD, J.; SCHAAPER, J. (2006). «Entre efficience, réactivité et apprentissage organisationnel - une étude qualitative sur le cas des filiales françaises en Chine». Management international, Vol.11, p.1-13.

Krueger, D. A.; Ding, B. (2009). «Ethical analysis and challenges of two international firms in China», Journal of business ethics, Vol. 89, $\mathrm{N}^{\circ}$ 2, p. 167-182.

Kwortnik, R.J. (2003). «Clarifying "Fuzzy" Hospitalitymanagement Problems with Depth Interviews and Qualitative Analysis», Cornell Hotel and Restaurant Administration Quartly, Vol. 44, N², p.117-129.

Lam, Simon S. K.; Yeung, Joseph C. K. (2010). «Staff localization and environmental uncertainty on firm performance in China », Asia Pacific Journal of Management, Vol. 27, Iss. 4, p. 677-695.

LAU, D. (2005). «China : Skills shortage makes long-term Talent Management key to success», Egon Zehnder International, Shanghai, Singapore and Kuala Lumpur.

LAW, K. S.; Wong, C.; WANG, K. D. (2004). «An empirical test of the model on managing the localization of human resources in the People's Republic of China », Int. J. of Human Resource Management, Vol. 15, N4, p.635-648.

Lyons, T. (2005). «Betting big on 'internationalising' local employees », China Staff, Vol.11, Iss. 5, p. 12-13.

Michael Page China (2008). Disponible sur http : //www. michaelpageinternational.com/webdav/site/mpi/shared/ Documents/PressReleases/China/ap_18_08_2008a.pdf, [Consulté le 15/06/2009]

MFE (Maison des français de l'étranger) (2010). «Marché du travail», Disponible sur http : //www.mfe.org/index.php/ Portails-Pays/Chine/Emploi-stage/Marche-du-travail, [Consulté le 06/02/2013]

MFE (Maison des français à l'étranger) (2011), «Chine», Disponible sur http : //www.mfe.org/index.php/Portails-Pays/ Chine, [Consulté le 06/02/2013]

MRIC Talent Report. (2013). MRIC Talent Report - Greater China Region and Singapore, Disponible sur http : //www. mric.asia/media/normal/199_2013_MRIC_Talent_Report_-_ Web_English_version__final_.pdf, [Consulté le 20/06/2014]

Negandhi, A.R.; Welge, M. (1984). Beyond Theory $Z$ : Global Rationalization Strategies of American, German and Japanese Multinational Companies, Advances in International Comparative Management, Greenwich, CA : JAI Press.

Prahalad, C. K.; Doz, Yves (1987). The Multinational Mission : Balancing Local Demands and Global Vision, London : The Free Press.
PWC Report. (2004). «Expatriate Localization Survey - Foreign Invested Enterprises in China Prefer Flexible Localization Programs », PriceWaterHouseCoopers Releases, Disponible sur www.pwccn.com/home/printeng/pr_091104.html, [Consulté le 16/01/2009]

PWC Report. (2009). «International Mobility Trends in Asia Hong Kong and China», March, Disponible sur http : //www. pwcias.com/home/eng/mobility_trends_hkcn_mar2009.html, [Consulté le 16/01/2012]

PWC Report. (2013). «Doing business and investing in China», Disponible sur http : //www.pwccn.com/webmedia/ doc/634940150734265198_iic_full.pdf, [Consulté le 27/06/2014].

QIU, L.; Chen, Y. (邱立成, 陈瑜). (2005). «Analysis on the management localization in the transnational corporations in China and its impact» (在华跨国公司管理本土化及其影 响分析). Journal of Beijing Administration Institute, Vol. 1, p.29-34.

Richards, L.; Rechards, T. (1994). «From filing cabinet to computer», dans A. Bryman \& R. G. Burgess (sous la direction de), Analysing qualitative data, London : Routledge. p. $146-172$.

Rogers, B. (1999). "The Expatriate in China - a Dying Species?», dans Localization Best Practice in China, Hong Kong : Euromoney Publications.

Selmer, J. (2003). Staff Localization and Organizational Characteristics : Western Business Operations in China, Asia Pacific, Business Review, Vol. 10, №1, p. 43-57.

SchaAper, J., Amann, B., Jaussaud, J., Nakamura, H., Miuzoguchi, S., (2012). «Human Resource Management in Asian Subsidiaries : comparison of French and Japanese MNCs», The International Journal of Human Resources Management, Vol.24, Iss. 7, p.1454-1470.

Symon, G.; Cassell, C. (Eds.). (2012). Qualitative organizational research : core methods and current challenges, Sage Publications.

WANG, G. (1993). «Greater China and the Chinese Overseas», The China Quarterly, Vol.136, p.926-948.

Wong, C.; LAW, K. S. (1999). «Managing Localization of Human Resources in the PRC : A Practical Model», Journal of World Business, Vol.34, $\mathrm{N}^{\circ} 1$, p. 26-40. 


\section{ANNEXE 1}

\section{Guide d'entretien}

Information sur l'entretien (nom de l'entreprise, lieu, date et durée de l'entretien, nom, fonction et parcours de l'interviewé)

Présentation générale de l'entreprise (activités, structures, ancienneté, effectif, etc.)

Bilan de postes occupés par différents types de cadres

Politiques et pratiques de la localisation (politiques, plan d'action, évolution et prévision de la localisation, etc.)

Est-ce qu'une localisation totale sans expatrié est envisageable et souhaitable? Si oui, pourquoi? Si non, pourquoi ? 


\section{ANNEXE 1 (SUITE)}

\section{Guide d'entretien}

Quels types de cadres chinois votre entreprise préfère-t-elle (chinois locaux, chinois retournés, chinois d'outre-mer)? Et pourquoi?

Quels sont vos ressentiments sur les politiques et les pratiques de la localisation de l'entreprise? Quelles sont les défis rencontrés ou potentiels?

Autres remarques 